University of Wollongong

Research Online

Faculty of Engineering and Information

Faculty of Engineering and Information

Sciences - Papers: Part B

Sciences

2019

Ultraviolet/persulfate pre-treatment for organic fouling mitigation of forward osmosis membrane: Possible application in nutrient mining from dairy wastewater

Biplob K. Pramanik

Royal Melbourne Institute of Technology, La Trobe University, biplobkp@uow.edu.au

Faisal I. Hai

University of Wollongong, faisal@uow.edu.au

Felicity Roddick

Royal Melbourne Institute of Technology

Follow this and additional works at: https://ro.uow.edu.au/eispapers1

Part of the Engineering Commons, and the Science and Technology Studies Commons

Research Online is the open access institutional repository for the University of Wollongong. For further information contact the UOW Library: research-pubs@uow.edu.au 


\title{
Ultraviolet/persulfate pre-treatment for organic fouling mitigation of forward osmosis membrane: Possible application in nutrient mining from dairy wastewater
}

\author{
Abstract \\ The forward osmosis (FO) membrane process has recently established its potential as an alternative \\ option to traditional membrane processes for producing clean water and recovering nutrients from \\ anaerobically treated wastewater streams. However, organic fouling of membrane leads to reduced flux, \\ and, thus, reduced amount of the extractable resources. In this study, the impact of ultraviolet/persulfate \\ (UV/PS) oxidation pre-treatment for the mitigation of organic fouling in the FO process during processing \\ of anaerobically treated dairy effluent (ATDE) was determined using a multi-cycle filtration method. The \\ UV/PS performance was compared with control pre-treatments such as stand-alone ultraviolet (UV) \\ irradiation and potassium persulfate (PS) oxidation. Size exclusion chromatography confirmed that flux \\ reduction over successive filtration cycles was due mainly to the humic substances and building blocks \\ i.e., sub-units of humic substances in the feedwater. Although all investigated pre-treatment options \\ mitigated membrane fouling, UV/PS achieved a greater enhancement in flux and decrease in both \\ reversible and irreversible foulant deposition than stand-alone UV and PS pre-treatments. This was \\ because UV/PS could generate sulfate and hydroxyl radicals, which were effective for decreasing the bulk \\ organic content and fluorescent organic content, and particularly for breaking down the large molecular \\ weight (MW) hydrophobic compounds to small MW hydrophilic components, resulting in less organics \\ adhesion to the membrane. This research shows the applicability of UV/PS pre-treatment for the organic \\ fouling mitigation of FO membrane during processing of ATDE for applications such as nutrient mining \\ from ATDE. \\ Disciplines \\ Engineering | Science and Technology Studies

\section{Publication Details} \\ Pramanik, B., Hai, F. I. \& Roddick, F. A. (2019). Ultraviolet/persulfate pre-treatment for organic fouling \\ mitigation of forward osmosis membrane: Possible application in nutrient mining from dairy wastewater. \\ Separation and Purification Technology, 217 215-220.
}

This journal article is available at Research Online: https://ro.uow.edu.au/eispapers1/2339 


\section{Research highlights}

- UV/PS led to a greater enhancement in flux than stand-alone UV and PS pretreatments

- UV/PS pre-treatment was particularly effective for reversible fouling mitigation

- Biopolymers and humics largely contributes to FO membrane reversible fouling

- UV led to greater removal of humics and building blocks than the PS treatment

- UV and PS had nearly same efficiency for biopolymers removal. 
Ultraviolet/persulfate pre-treatment for organic fouling mitigation of forward osmosis membrane: possible application in nutrient mining from dairy wastewater

\section{Biplob Kumar Pramanik ${ }^{\text {a,c*}}$, Faisal I Hai ${ }^{\text {, }}$ Felicity A. Roddick ${ }^{c}$}

${ }^{\mathrm{a}}$ Department of Engineering \& Mathematic Sciences, LaTrobe University, Bendigo, VIC3552, Australia

${ }^{\mathrm{b}}$ Strategic Water Infrastructure Laboratory, School of Civil, Mining and Environmental Engineering, University of Wollongong, Wollongong, NSW 2522, Australia

${ }^{\text {c}}$ School of Engineering, RMIT University, Melbourne, VIC 3000, Australia

Corresponding author Email: b.pramanik@latrobe.edu.au, biplob_02ce@yahoo.com; Tel: +6135444 7154, 


\section{Abstract}

The forward osmosis (FO) membrane process has recently established its potential as an alternative option to traditional membrane processes for producing clean water and recovering nutrients from anaerobically treated wastewater streams. However, organic fouling of membrane leads to reduced flux, and, thus, reduced amount of the extractable resources. In this study, the impact of ultraviolet/persulfate (UV/PS) oxidation pre-treatment for the mitigation of organic fouling in the FO process during processing of anaerobically treated dairy effluent (ATDE) was determined using a multi-cycle filtration method. The UV/PS performance was compared with control pre-treatments such as stand-alone ultraviolet (UV) irradiation and potassium persulfate (PS) oxidation. Size exclusion chromatography confirmed that flux reduction over successive filtration cycles was due mainly to the humic substances and building blocks i.e., sub-units of humic substances in the feedwater. Although all investigated pre-treatment options mitigated membrane fouling, UV/PS achieved a greater enhancement in flux and decrease in both reversible and irreversible foulant deposition than stand-alone UV and PS pre-treatments. This was because UV/PS could generate sulfate and hydroxyl radicals, which were effective for decreasing the bulk organic content and fluorescent organic content, and particularly for breaking down the large molecular weight (MW) hydrophobic compounds to small MW hydrophilic components, resulting in less organics adhesion to the membrane. This research shows the applicability of UV/PS pre-treatment for the organic fouling mitigation of FO membrane during processing of ATDE for applications such as nutrient mining from ATDE.

Keywords: Anaerobically treated dairy effluent, forward osmosis, organic fouling, ultraviolet/persulfate. 


\section{Introduction}

Due to the rising concern regarding freshwater shortage, membrane technologies have been broadly implemented for drinking water, wastewater and seawater treatment to augment the water supply (Elimelech \& Phillip, 2011). Over the past decade, there has been increasing emphasis on the osmotically driven forward osmosis (FO) membrane process for municipal wastewater (Cath et al., 2006) and industrial wastewater treatment (Holloway et al., 2007), and seawater desalination (Phuntsho et al., 2014). The key benefit of the FO process is high contaminants rejection without requiring high hydraulic pressure for system operation (Cath et al., 2006). This process, among other applications, can concentrate low-strength wastewater and thus enrich the wastewater-derived nutrients, which can be used as a fertiliser for agriculture (Xue et al., 2015).

It has been reported that the osmotically-driven FO process has a lower propensity for fouling than pressure-driven membrane processes (Kim et al., 2014). However, FO membrane fouling does occur, especially when the feedwater contains a high concentration of organic matter (Attarde et al., 2017; Bar-Zeev \& Elimelech, 2014; Jeong et al., 2013). Available studies on the causes/mechanisms of organic fouling in the FO process have predominantly used synthetic test solutions (Heo et al., 2016; Kim et al., 2015; Mi \& Elimelech, 2010). However, control of organic fouling for this emerging technology for nutrient recovery from high strength real wastewater has been overlooked.

Pre-treatment of feedwater before membrane filtration is a typical process to alleviate pressure-driven membrane fouling caused by organic matter. Removal of organics and changes in the structure of organic content are the dominant mechanisms involved in fouling mitigation with feedwater pre-treatment (Huang et al., 2009). Several processes such as 
chemical, biological and advanced oxidation have been applied as a pre-treatment for membrane filtration to remove the organic foulants from feedwater (Huang et al., 2009). Coagulation pre-treatment is widely used for fouling control (Dong et al., 2007); however, this process produces chemical sludge which must be managed appropriately. Conversely, Pramanik et al. (2016) reported that flux improvement for the microfiltration (MF) of secondary effluent reduced with the service time of the biologically activated carbon filtration pre-treatment. This was because of the gradually decreased removal of a major foulant (i.e., humic substances) along with the gradual decline in adsorption on activated carbon.

Advanced oxidation processes (AOPs) present an emerging option for degradation of organics from water as well as high strength wastewater. The key mechanism of AOPs is the production of hydroxyl radicals $\left(\mathrm{HO}^{\circ}\right)$ via mechanical and electrical processes (Comninellis et al., 2008; Parsons, 2004). Hydroxyl radicals can be generated with standalone oxidants or integration of ultraviolet (UV) radiation, ozone and hydrogen peroxide $\left(\mathrm{H}_{2} \mathrm{O}_{2}\right)$ (Legrini et al., 1993; Wang et al., 2000). Studies have demonstrated the potential of UV irradiation as pretreatments for reducing organic matter, and thus, reduced organic fouling in MF systems for drinking water treatment (Malek et al., 2006a; Malek et al., 2006b). UV/ $\mathrm{H}_{2} \mathrm{O}_{2}$ feed pretreatment could notably reduce the organic fouling of a MF membrane (Zhang et al., 2015). The fouling reduction was primarily due to change in molecular structure or degradation of macromolecules into smaller organic molecules (Song et al., 2008). Song et al. (2004) noted that the $\mathrm{UV} / \mathrm{H}_{2} \mathrm{O}_{2}$ pre-treatment could distinctly improve the performance of nanofiltration process during ground water treatment due to removal of organics, hydrogen sulfide and alachlor. Recently, ultraviolet/persulfate (UV/PS)-based AOPs have been explored for this purpose (Zhang et al., 2016). In this process, reactive radicals such as sulfate $\left(\mathrm{SO}_{4}{ }^{\circ}\right)$ and $\mathrm{HO}^{\circ}$ which have a high reaction affinity with organic matter, are generated via Eq. (1)-(3) when 
PS is subjected to UV irradiation (Yang et al., 2017; Zhang et al., 2016). The UV/PS process can degrade organics efficiently (Liu et al., 2017; Lou et al., 2016). Xiao et al. (2016) reported that UV/PS may be more cost-effective than the $\mathrm{UV} / \mathrm{H}_{2} \mathrm{O}_{2}$ process based on the electrical energy and chemical cost. They also found that UV/PS was more effective in mineralizing organic compounds. Hence, it is likely that UV/PS pre-treatment would contribute to the control of organic fouling during nutrient recovery from anaerobically treated dairy effluent (ATDE) treatment using FO. There are no references in the literature regarding the effect of UV/PS pre-treatment of a high strength wastewater such as ATDE on FO fouling mitigation.

$$
\begin{array}{ll}
\mathrm{S}_{2} \mathrm{O}_{8}^{2-} \stackrel{U V}{\longrightarrow} 2 \mathrm{SO}_{4}^{-}-\varnothing=1.4 \\
\mathrm{SO}_{4}^{\cdot-}+\mathrm{OH}^{-} \rightarrow \mathrm{SO}_{4}^{2-}+\mathrm{HO}^{\circ} \quad k=6.5 \times 10^{7} \mathrm{M}^{-1} \mathrm{~s}^{-1} \\
\mathrm{SO}_{4}^{--}+\mathrm{H}_{2} \mathrm{O} \rightarrow \mathrm{HSO}_{4}^{2-}+\mathrm{HO}^{\cdot} \quad k=8.3 \mathrm{M}^{-1} \mathrm{~S}^{-1}
\end{array}
$$

The aim of this research was to explore the effect of ATDE pretreatment by UV/PS, standalone UV and PS on mitigation of organic fouling of a FO membrane using a multi-cycle filtration protocol. Mass balance was conducted to elucidate the major components responsible for FO fouling. The effect of the pre-treatments on nutrient removal/recovery was also determined.

\section{Materials and methods}

\subsection{Feed solution}

The anaerobically treated dairy effluent was collected from a commercial dairy farm in Gerringong, Wollongong, Australia. At that site, the dairy wastewater is treated by passing it 
through anaerobic and then aerobic ponds. Then the biologically treated effluent is released to the sewer network for further treatment at a municipal wastewater treatment plant. Samples were collected from the anaerobic ponds and kept at $4{ }^{\circ} \mathrm{C}$ until use. Before all tests, the effluent was brought to room temperature $\left(22 \pm 2{ }^{\circ} \mathrm{C}\right)$.

\subsection{Pre-treatment test protocol}

\subsubsection{Potassium persulfate}

Reagent grade potassium persulfate $\left(\mathrm{K}_{2} \mathrm{~S}_{2} \mathrm{O}_{8}\right)$ was purchased from Sigma Aldrich (Australia). Experiments were conducted in a $500 \mathrm{~mL}$ conical flask at laboratory temperature $\left(22 \pm 2{ }^{\circ} \mathrm{C}\right)$.

A range of PS doses was tested (1.0 $\mathrm{mM}$ to $10 \mathrm{mM})$ for DOC reduction from ATDE, and the optimum PS dose was 5 mM. Following the protocol of Cheng et al. (2017a), the sample was rapidly mixed for $1 \mathrm{~min}$ at $200 \mathrm{rpm}$, after that subjected to slow mixing for $20 \mathrm{~min}$ at $50 \mathrm{rpm}$ in a rotary shaker, and then the pre-treated water samples were used for FO tests.

\subsubsection{UV and UV/PS}

A laboratory-scale UV photo-oxidation system was purchased from Ace Glass (NJ, USA). The system consisted of two cylindrical reactors (inner and outer) and a low-pressure UV lamp. The inner reactor, which contained the UV lamp, was made of quartz and had a diameter of $20 \mathrm{~cm}$ and a height of $50 \mathrm{~cm}$. The $27 \mathrm{~cm}$-long UV lamp emitted light at $254 \mathrm{~nm}$ with a UV energy of $83 \mathrm{~W}$ and intensity of $1.04 \mathrm{Wcm}^{-2}$. The temperature of this reactor was maintained at $20^{\circ} \mathrm{C}$ by recirculating cool water through the ports attached. The test solution in the outer reactor was mixed well with a magnetic stirrer. An UV irradiation time of 30 min was used in this study as suggested by Umar et al. (2014), who noted that the reduction 
of organics followed a pseudo first order reaction for 30 min when majority of the organics was removed.

For the UV/PS system, $5 \mathrm{mM}$ PS was mixed with the dairy effluent before irradiating it for 30 min. A dose of $\mathrm{Na}_{2} \mathrm{~S}_{2} \mathrm{O}_{3}$ stoichiometrically equivalent to the added PS concentration (Tian et al., 2018) was added to pre-treated samples after sampling to quench the residual oxidants.

\subsection{Experimental protocol of the FO system}

A lab-scale cross flow FO setup with a filtration area of $50 \mathrm{~cm}^{2}$ was used. A diagram of the FO set-up is given in Figure 1. Flat sheet thin film composite (TFC) membranes were purchased from Porifera Inc, Court Hayward, USA. The membrane was made of polyamide on polysulfone support. Key properties of the TFC membrane are shown in Supplementary Table S1. The FO membrane cell comprised two identical blocks (feed side and draw solution side) made of acrylic plastic. Channels of effective dimensions of $36 \mathrm{~mm}$ width, $167 \mathrm{~mm}$ length and $1.4 \mathrm{~mm}$ height were engraved on both sides for the feed and draw solutions.

\section{Figure 1}

The volume of the feed and draw solutions was $1 \mathrm{~L}$. The solutions were recirculated at 1 L/min by two gear pumps (Micropump, Washington, USA) regulated by rotameters. NaCl (1 M, analytical grade) was used as the draw solution. The draw solution tank was positioned on an electronic balance (Mettler-Toledo Inc., New Jersey, USA) to record the weight increment of the permeate for computing water flux. A concentrated $\mathrm{NaCl}$ solution (5 M) was used for 
maintaining constant osmotic pressure in the diluted draw solution tank. A conductivity probe (Cole-Parmer, Illinois, USA) was submerged into the draw solution. This probe was linked to the NaCl-dosing peristaltic pump to control the draw solution concentration when the diluted draw solution conductivity dropped below the set point $(62.2 \mathrm{mS} / \mathrm{cm})$.

Before the filtration test, each membrane was submerged into Milli-Q water for 30 min for eliminating residual preservative reagents. Following this, the clean water flux of the membrane was measured using Milli Q water. Each FO filtration experiment comprised three filtration cycles, and a fresh feed solution was used for every fouling experiment. After every cycle, the used membrane was osmotically backwashed for 30 min (Yu et al., 2017). This was conducted by changing the draw solution with Milli-Q water. In the backwashing process, draw solution (0.5 $\mathrm{M} \mathrm{NaCl})$ was used as a feed solution. When Milli-Q water flows through the draw side channel, the osmotic pressure gradients are formed in the opposite direction, and permeate (i.e. backwash water) flows from the draw (Milli-Q water) to feed sides. Therefore, foulants on the membrane surface are detached by this reverse flow. The backwash water was tested for identifying and quantifying the organic molecules responsible to reversible and irreversible fouling. In this study, organics released from membranes were termed as reversible foulant. The remaining organics attached on the membrane surface were termed as irreversible foulant. After completing the backwashing process, Milli-Q water was filtered through the membrane to enable determination of the water recovery (i.e., fouling reversibility).

\subsection{Analytical methods}

Total phosphate (TP) and total nitrogen (TN) were determined using Hach reagents and a DR3900 spectrophotometer. Dissolved organic carbon (DOC) was determined using a 
Sievers 820 TOC analyser. The ultraviolet absorbance at $254 \mathrm{~nm}\left(\mathrm{UV}_{254}\right)$ was measured using a Unicam UV2 spectrophotometer. Before these analyses, water samples were filtered $(0.45 \mu \mathrm{m})$. Electrical conductivity and $\mathrm{pH}$ of the solution were determined by an Orion 4-Star Plus $\mathrm{pH} /$ conductivity meter (Thermo Scientific, MA). The turbidity of the samples was determined by a turbidity meter (Hach, 2100P).

Fluorescent organics were determined by fluorescence excitation-emission matrix (EEM) spectrometer (LS55, PerkinElmer) over excitation and emission wavelength ranges of 220$465 \mathrm{~nm}$ and 280-550 nm, respectively. The band width for both excitation and emission was set at $5 \mathrm{~nm}$. The excitation and emission slits were maintained at $7 \mathrm{~nm}$ and the scanning speed was set at $700 \mathrm{~nm} / \mathrm{min}$. The molecular weight distribution of the organics was determined by liquid chromatography with organic carbon detection (LC-OCD) (Model 8, DOC-Labor). The LC unit separates organic compounds according to molecular size and the separated compounds are detected by online detectors. Molar mass calibration of the column is conducted by using reference of humic acid and fulvic acid standards. The details of the fluorimetric and LC-OCD spectra analysis have been described elsewhere (Pramanik et al., 2016). Each sample was diluted and filtered $(0.45 \mu \mathrm{m})$ before fluorimetric and LC-OCD analysis.

\section{Results and Discussion}

\subsection{Water quality}

The characteristics of the untreated and treated ATDE are presented in Table 1. There was a higher reduction of DOC by UV irradiation (15\%) than by PS (10\%). Notably, UV can break carbon-carbon bonds and aromatic structures and it can also induce partial mineralisation of the organic matter (Puspita et al., 2011; Tian et al., 2018). The reduction of $\mathrm{UV}_{254}$ was more prominent but showed a similar pattern to that of DOC reduction. UV/PS pre-treatment led to 
markedly higher removal efficiency of DOC and $\mathrm{UV}_{254}$ absorbance than for stand-alone UV or PS treatment. This shows that the presence of persulfate during UV irradiation improved organics degradation. This was likely due to the enhanced generation of hydroxyl and sulfate radicals which could degrade unsaturated organic fractions followed by the resistant fractions of the organics, when persulfate was exposed to UV radiation (Cheng et al., 2017b). The value of specific ultraviolet absorbance (SUVA) designates the aromatic compounds of organics. The value of SUVA) for the ATDE was $3.27 \mathrm{~L} / \mathrm{m} \cdot \mathrm{mg}$, representing moderately aromatic organics (Ghernaout, 2014). PS, UV and UV/PS treatment reduced the SUVA by 9.5\%, $12.5 \%$ and $17.2 \%$, respectively. These reductions in SUVA implied a greater breakdown of humics, and thus, the organics were converted to less aromatic components by all processes.

There was minimal removal of nutrient species after the treatment processes. The reduction in TN and TP was $8-10 \%$ and $4-6 \%$ by PS and UV treatment, respectively. The reduction in TP was marginally higher for the UV/PS than the stand-alone PS and UV treatments. Since these processes led to only low levels of nutrient reduction, they can be used for membrane fouling reduction in the FO process during 'nutrient recovery' from ATDE.

\section{Table 1}

\subsection{FO flux in multi-cycle filtration tests}

The normalised water flux (J/Jo) vs permeate volume $(\mathrm{V})$ for various pre-treated ATDE samples are shown in Figure 2a. Untreated ATDE resulted in a significant flux decline: the flux declined quickly within the first $60 \mathrm{~min}$. A permeate volume of $200 \mathrm{~mL}$ could be withdrawn within 495 min. UV pre-treatment performed better than the PS treatment for 
improving the water flux, with the same permeate volume of $200 \mathrm{~mL}$ obtained after $325 \mathrm{~min}$ for UV and 430 min for PS. The flux was substantially increased for the UV/PS-treated ATDE sample due to the significant removal and breakdown of the organic matter. Permeate flux for successive filtration cycles continuously decreased for the PS, UV and UV/PS treated samples due to the increase of hydraulic resistance resulting from organic fouling. This result can be ascribed to the change of concentration polarisation due to the formation of fouling layer (Kim et al., 2012).

During osmotic backwashing, water moves in the reverse direction from the draw-to-feed side, and this can dislodge the organic fouling layer on membrane. In this study, osmotic backwashing was unable to restore the FO performance completely, and the flux recovery rate decreased with successive filtration cycles for both the untreated and treated ATDE samples (Figure 2b). However, the water flux recovery was the highest for UV/PS treated ATDE samples. For example, after the backwash following the first filtration cycle, there was a water recovery of 93\%, 94\% and 98\% for PS, UV and UV/PS treated ATDE samples, respectively (Figure 2b).

\section{Figure 2}

\subsection{Characterisation of organic foulants using fluorescence EEM spectra and size exclusion chromatography}

\subsubsection{Fluorescence EEM spectra}

Fluorescence EEM spectra were used to explain the pre-treatment role on the characteristics of the fluorescent organic molecules (Chen et al., 2003). An EEM spectrum comprises five sections (Figure 3a). Section I is related to tyrosine-based aromatic proteins (AP) and section II is related to tryptophan-based AP. Section III is associated with the fluorescence response 
of fulvic acid (FA)-like materials and section IV represents the fluorescence response of soluble microbial products (SMPs). Section V is related with humic acid (HA)-like materials. The untreated ATDE exhibited the following fluorescence intensity for each molecule: HAlike $>$ FA-like $>$ SMPs $>$ AP

The fluorescence regional integration technique (Chen et al., 2003) was applied to interpret the EEMs (Figure 3b) for quantifying the changes in the fluorescent organics after the pretreatments of the ATDE. A complete rejection of the fluorescent components by the FO membrane was observed, indicating the likely role of these fluorescent substances in the FO fouling. All three pre-treatments were able to reduce fluorescence in all five sections, demonstrating the loss of aromaticity of the organic component (Uyguner \& Bekbolet, 2005), but at different rates. Consistent with the $\mathrm{UV}_{254}$ reductions (Table 1), UV/PS achieved more reduction in the fluorescent organics than the stand-alone UV and PS treatments (Figure 3b), and the fluorescent components were more susceptible to standalone UV than the stand-alone PS treatment. UV/PS specially degraded the HA-like and FA-like substances. A comparable result was stated by Puspita et al. (2011) who found that $\mathrm{UV} / \mathrm{H}_{2} \mathrm{O}_{2}$ oxidation preferentially degraded humic organics over other fluorescent molecules. It is noteworthy that the extent of fluorescence reduction was significantly greater than for DOC and $\mathrm{UV}_{254}$. Puspita et al. (2011) also confirmed higher degradation of fluorescent organics compared to total DOC during $\mathrm{UV} / \mathrm{H}_{2} \mathrm{O}_{2}$ oxidation of dissolved organic matter in secondary effluent. This is because fluorescent molecules generally have high UV absorbance and, thus, are more susceptible to UV radiation than non-fluorescent molecules (Kavurmaci \& Bekbolet, 2014; Phong \& Hur, 2015). Cho and Choi (2002) also noted that the faster degradation of fluorescent components is attributable to the fluorescence resulting from the $\pi^{*}-\pi$ changes in organics along with its rapid destruction under UV irradiation. Because of the effective degradation of the large 
humic-like substances, UV/PS pre-treatment could effectively reduce membrane fouling during ATDE treatment for nutrient recovery using the FO process.

\section{Figure 3}

\subsubsection{Size exclusion chromatography}

LC-OCD analysis revealed the influence of pre-treatment on the apparent molecular weight (MW) distribution of the organics. Based on the molecular weight, the organic compounds were separated into five fractions (Figure 4a). These were biopolymers, humic substances, building blocks i.e., sub-units of humic substances, low MW acids, and low MW neutrals (Huber et al., 2011). The proportions to the total DOC for the untreated samples were 8.5\% (biopolymers), 50\% (humic substances), 16.5\% (building blocks), and 22.5\% (low MW organics i.e., acids and neutrals). It was found that there was no passage of biopolymers, humic substances or building blocks through the FO membrane from the feed to the draw solution (not shown), indicating that these molecules can lead to fouling. Over $90 \%$ of the low MW organics was also retained by the FO membrane, and, thus, are also likely to contribute to fouling resistance.

UV pre-treatment achieved greater reduction of humics and building blocks than the PS treatment although both had nearly same efficiency for biopolymers removal (Figure 4b). The reduction in these substances was consistent with the reduction in $\mathrm{UV}_{254}$ (Table 1). Both standalone PS and UV were efficient in removing UV-absorbing compounds (humic substances and building blocks), but not biopolymers. UV treatment led to minimal removal of low MW organics whereas PS did not remove these molecules. This is because UV irradiation can mineralise low MW organic molecules marginally (Van Geluwe et al., 2011). 
Studies have reported that the low MW fraction of natural organic matter was the most onerous to remove, because majority components in this portion were reluctant to the oxidative processes (Buchanan et al., 2005). Another explanation could be that the concentration of smaller MW organics was increased due to breakdown of the humics and building blocks. This counterbalanced the loss of low MW molecules by mineralisation, thus resulting in a consistent total concentration of low MW organics after pre-treatments. Compared to stand-alone UV and PS treatments, UV/PS gave significantly higher reductions in biopolymers, humics and building blocks. This pre-treatment degraded high- and mediumMW hydrophobic compounds into low MW hydrophilic molecules, resulting in less build-up of organics on the membrane surface by decreasing the adhesion force between the organics and the membrane. A similar finding was noted by Puspita et al. (2011) and Zhang et al. (2015) who found that $\mathrm{UV} / \mathrm{H}_{2} \mathrm{O}_{2}$ could degrade humic acids and the hydrophobic fraction, resulting in the generation of some hydrophilic components. It is likely that the size of some of these hydrophilic molecules are smaller than the membrane pores and thus can pass through the FO membrane, and consequently do not cause permeate flux reduction.

\section{Figure 4}

\subsection{Organic foulants distribution on the FO membrane}

Applying the concept of mass balance, the distributions of the different organic components were established to understand their fate in the filtration of the untreated and pre-treated ATDE samples (Figure 5). The designations 'reversibly deposited' and 'irreversibly deposited' foulants denote to the organic matter in the backwash water and residual attached in/on the FO membrane, respectively. As mentioned in Sections 3.3.1 and 3.3.2, both fluorescent and non-fluorescent molecules (except low MW compounds of which only 8\% 
were transported across the membrane but which contained $22.5 \%$ of the total DOC), were not transported through the membrane to the draw solution. Thus, the mass balance calculation mainly accounted for the deposition of organics on the membrane surface, either reversibly or irreversibly.

Both untreated and treated ATDE led to greater deposition of reversible foulants than irreversible foulants. The biopolymers and humic substances mainly accumulated on the active layer of the membrane surface due to size exclusion, then formed a cake layer and, hence, reduced the flux, indicating that these molecules largely contributes to the reversible fouling of the FO membrane. On the other hand, building blocks were strongly attached to the membrane surface due to the hydrophobic nature of these molecules. All three pretreatments decreased the deposition of reversible and irreversible foulants (in terms of biopolymers, humics, building blocks and low MW organics) on the membrane surface, but to different levels. There was a greater reversible and irreversible foulants reduction by UV/PS than by stand-alone UV, which in turn was higher than for the stand-alone PS treatment. This demonstrated that the UV/PS pre-treatment exhibited good performance in improving water flux by reducing the deposition of both reversible and irreversible foulant. It was noted that the deposition of reversible foulant was lower than irreversible foulant after the pre-treatments.

The deposition of both reversible and irreversible foulant increased with multi-cycles for both untreated and treated ATDE samples. This was likely due to the initial organics deposition on the membrane surface and then further deposition and aggregation of the organics over successive cycles. Humic substances and building blocks contributed more than the biopolymers and low MW organics to the irreversibly attached foulant during successive 
filtration cycles. On the other hand, the biopolymer and low MW organics contributed more to the reversible than the irreversible foulant over the multi cycles, while some small low MW molecules possibly deposited into the membrane pores and thus could also have contributed to irreversible fouling.

\section{Figure 5}

\section{Conclusions}

This study investigated the effect of ultraviolet/persulfate (UV/PS) process as a pre-treatment for mitigation of organic fouling of FO membrane during ATDE treatment using a multicycle filtration method. The UV/PS treatment performance was compared with standalone UV and PS as control pre-treatments. It was noted that all pre-treatments could reduce membrane fouling, but the fouling control performance was significantly higher for UV/PS. Characterisation of both untreated and treated ATDE samples using size exclusion chromatography showed that the contribution of large MW biopolymers and humic substances to the hydraulically reversible fouling was higher than that of the building block and low MW organics. This was due to the size exclusion effect. The increase in the deposition of irreversible foulants with multi-cycles was due mostly to the humic substances, building block and low MW organics of the feedwater. These molecules were initially deposited followed by further deposition and aggregation on the membrane. UV/PS pretreatment achieved the best reduction of the deposition of both reversibly deposited and irreversibly deposited foulants on the membrane than PS or UV treatment. This was mainly due to the better removal of the organics and breakdown of the high MW organics to low MW organics. 
This study demonstrated that UV/PS is a potentially promising pre-treatment for reducing organic fouling of FO during nutrient recovery from ATDE. This treatment could reduce the operating costs by maintaining an economic flux rate. Further work to explore the long-term operation of FO on the filterability of ATDE is required. Moreover, understanding the products formed by the UV/PS advanced oxidation process during nutrient recovery from the ATDE using the FO system may be worth considering.

\section{Acknowledgements}

A Vice-Chancellor's Postdoctoral Research Fellowship to Biplob Kumar Pramanik by the University of Wollongong, Australia is acknowledged. The authors would also like to thank Mrs Khorshed Chinu (University of New South Wales, Australia) for analysing the LC-OCD samples.

\section{References}

Attarde, D., Jain, M., Singh, P.K., Gupta, S.K. 2017. Energy-efficient seawater desalination and wastewater treatment using osmotically driven membrane processes. Desalination, 413, 86-100.

Bar-Zeev, E., Elimelech, M. 2014. Reverse osmosis biofilm dispersal by osmotic backflushing: cleaning via substratum perforation. Environmental Science \& Technology Letters, 1(2), 162-166.

Buchanan, W., Roddick, F., Porter, N., Drikas, M. 2005. Fractionation of UV and VUV pretreated natural organic matter from drinking water. Environmental Science \& Technology, 39(12), 4647-4654.

Cath, T.Y., Childress, A.E., Elimelech, M. 2006. Forward osmosis: principles, applications, and recent developments. Journal of Membrane Science, 281(1), 70-87. 
Chen, W., Westerhoff, P., Leenheer, J.A., Booksh, K. 2003. Fluorescence excitationemission matrix regional integration to quantify spectra for dissolved organic matter. Environmental Science \& Technology, 37(24), 5701-5710.

Cheng, X., Liang, H., Ding, A., Tang, X., Liu, B., Zhu, X., Gan, Z., Wu, D., Li, G. 2017a. Ferrous iron/peroxymonosulfate oxidation as a pretreatment for ceramic ultrafiltration membrane: Control of natural organic matter fouling and degradation of atrazine. Water Research, 113, 32-41.

Cheng, X., Liang, H., Ding, A., Zhu, X., Tang, X., Gan, Z., Xing, J., Wu, D., Li, G. 2017b. Application of Fe (II)/peroxymonosulfate for improving ultrafiltration membrane performance in surface water treatment: Comparison with coagulation and ozonation. Water Research, 124, 298-307.

Cho, Y., Choi, W. 2002. Visible light-induced reactions of humic acids on TiO2. Journal of Photochemistry and Photobiology A: Chemistry, 148(1-3), 129-135.

Comninellis, C., Kapalka, A., Malato, S., Parsons, S.A., Poulios, I., Mantzavinos, D. 2008. Advanced oxidation processes for water treatment: advances and trends for R\&D. Journal of Chemical Technology and Biotechnology, 83(6), 769-776.

Dong, B.-Z., Yan, C., Gao, N.-y., Fan, J.-c. 2007. Effect of coagulation pretreatment on the fouling of ultrafiltration membrane. Journal of Environmental Sciences, 19(3), 278283.

Elimelech, M., Phillip, W.A. 2011. The future of seawater desalination: energy, technology, and the environment. Science, 333(6043), 712-717.

Ghernaout, D. 2014. The hydrophilic/hydrophobic ratio vs. dissolved organics removal by coagulation-A review. Journal of King Saud University-Science, 26(3), 169-180. 
Heo, J., Chu, K.H., Her, N., Im, J., Park, Y.-G., Cho, J., Sarp, S., Jang, A., Jang, M., Yoon, Y. 2016. Organic fouling and reverse solute selectivity in forward osmosis: Role of working temperature and inorganic draw solutions. Desalination, 389, 162-170.

Holloway, R.W., Childress, A.E., Dennett, K.E., Cath, T.Y. 2007. Forward osmosis for concentration of anaerobic digester centrate. Water Research, 41(17), 4005-4014.

Huang, H., Schwab, K., Jacangelo, J.G. 2009. Pretreatment for low pressure membranes in water treatment: a review. Environmental Science \& Technology, 43(9), 3011-3019.

Huber, S.A., Balz, A., Abert, M., Pronk, W. 2011. Characterisation of aquatic humic and non-humic matter with size-exclusion chromatography-organic carbon detectionorganic nitrogen detection (LC-OCD-OND). Water Research, 45(2), 879-885.

Jeong, S., Naidu, G., Vigneswaran, S. 2013. Submerged membrane adsorption bioreactor as a pretreatment in seawater desalination for biofouling control. Bioresource Technology, 141, 57-64.

Kavurmaci, S.S., Bekbolet, M. 2014. Tracing TiO2 photocatalytic degradation of humic acid in the presence of clay particles by excitation-emission matrix (EEM) fluorescence spectra. Journal of Photochemistry and Photobiology A: Chemistry, 282, 53-61.

Kim, C., Lee, S., Hong, S. 2012. Application of osmotic backwashing in forward osmosis: mechanisms and factors involved. Desalination and Water Treatment, 43(1-3), 314322.

Kim, Y., Elimelech, M., Shon, H.K., Hong, S. 2014. Combined organic and colloidal fouling in forward osmosis: Fouling reversibility and the role of applied pressure. Journal of Membrane Science, 460, 206-212.

Kim, Y., Lee, S., Shon, H.K., Hong, S. 2015. Organic fouling mechanisms in forward osmosis membrane process under elevated feed and draw solution temperatures. Desalination, 355, 169-177. 
Legrini, O., Oliveros, E., Braun, A. 1993. Photochemical processes for water treatment. Chemical Reviews, 93(2), 671-698.

Liu, B., Qu, F., Chen, W., Liang, H., Wang, T., Cheng, X., Yu, H., Li, G., Van der Bruggen, B. 2017. Microcystis aeruginosa-laden water treatment using enhanced coagulation by persulfate/Fe (II), ozone and permanganate: Comparison of the simultaneous and successive oxidant dosing strategy. Water Research, 125, 72-80.

Lou, X., Xiao, D., Fang, C., Wang, Z., Liu, J., Guo, Y., Lu, S. 2016. Comparison of UV/hydrogen peroxide and UV/peroxydisulfate processes for the degradation of humic acid in the presence of halide ions. Environmental Science and Pollution Research, 23(5), 4778-4785.

Malek, F., Harris, J., Roddick, F. 2006a. Interrelationship of photooxidation and microfiltration in drinking water treatment. Journal of Membrane Science, 281(1-2), 541-547.

Malek, F., Harris, J., Roddick, F. 2006b. Photooxidative pretreatment to improve sustainable operation of the microfiltration of drinking water. Developments in Chemical Engineering and Mineral Processing, 14(1 $\quad-226219$

Mi, B., Elimelech, M. 2010. Organic fouling of forward osmosis membranes: fouling reversibility and cleaning without chemical reagents. Journal of Membrane Science, 348(1-2), 337-345.

Parsons, S. 2004. Advanced oxidation processes for water and wastewater treatment. IWA publishing.

Phong, D.D., Hur, J. 2015. Insight into photocatalytic degradation of dissolved organic matter in UVA/TiO2 systems revealed by fluorescence EEM-PARAFAC. Water Research, 87, 119-126. 
Phuntsho, S., Lotfi, F., Hong, S., Shaffer, D.L., Elimelech, M., Shon, H.K. 2014. Membrane scaling and flux decline during fertiliser-drawn forward osmosis desalination of brackish groundwater. Water Research, 57, 172-182.

Pramanik, B.K., Roddick, F.A., Fan, L. 2016. Long-term operation of biological activated carbon pre-treatment for microfiltration of secondary effluent: correlation between the organic foulants and fouling potential. Water Research, 90, 405-414.

Puspita, P., Roddick, F., Porter, N. 2011. Decolourisation of secondary effluent by UVmediated processes. Chemical Engineering Journal, 171(2), 464-473.

Song, W., Ravindran, V., Koel, B.E., Pirbazari, M. 2004. Nanofiltration of natural organic matter with H2O2/UV pretreatment: fouling mitigation and membrane surface characterization. Journal of Membrane Science, 241(1), 143-160.

Song, W., Ravindran, V., Pirbazari, M. 2008. Process optimization using a kinetic model for the ultraviolet radiation-hydrogen peroxide decomposition of natural and synthetic organic compounds in groundwater. Chemical Engineering Science, 63(12), 32493270.

Tian, J., Wu, C., Yu, H., Gao, S., Li, G., Cui, F., Qu, F. 2018. Applying ultraviolet/persulfate (UV/PS) pre-oxidation for controlling ultrafiltration membrane fouling by natural organic matter (NOM) in surface water. Water Research, 132, 190-199.

Umar, M., Roddick, F., Fan, L. 2014. Effect of coagulation on treatment of municipal wastewater reverse osmosis concentrate by UVC/H2O2. Journal of Hazardous Materials, 266, 10-18.

Uyguner, C.S., Bekbolet, M. 2005. Evaluation of humic acid photocatalytic degradation by UV-vis and fluorescence spectroscopy. Catalysis Today, 101(3-4), 267-274. 
Van Geluwe, S., Braeken, L., Van der Bruggen, B. 2011. Ozone oxidation for the alleviation of membrane fouling by natural organic matter: A review. Water Research, 45(12), 3551-3570.

Wang, G.-S., Hsieh, S.-T., Hong, C.-S. 2000. Destruction of humic acid in water by UV light—catalyzed oxidation with hydrogen peroxide. Water Research, 34(15), 38823887.

Xiao, Y., Zhang, L., Zhang, W., Lim, K.-Y., Webster, R.D., Lim, T.-T. 2016. Comparative evaluation of iodoacids removal by UV/persulfate and UV/H2O2 processes. Water Research, 102, 629-639.

Xue, W., Tobino, T., Nakajima, F., Yamamoto, K. 2015. Seawater-driven forward osmosis for enriching nitrogen and phosphorous in treated municipal wastewater: effect of membrane properties and feed solution chemistry. Water Research, 69, 120-130.

Yang, Y., Lu, X., Jiang, J., Ma, J., Liu, G., Cao, Y., Liu, W., Li, J., Pang, S., Kong, X. 2017. Degradation of sulfamethoxazole by UV, UV/H2O2 and UV/persulfate (PDS): formation of oxidation products and effect of bicarbonate. Water Research, 118, 196207.

Yu, Y., Lee, S., Maeng, S.K. 2017. Forward osmosis membrane fouling and cleaning for wastewater reuse. Journal of Water Reuse and Desalination, 7(2), 111-120.

Zhang, X., Fan, L., Roddick, F.A. 2015. Effect of feedwater pre-treatment using UV/H2O2 for mitigating the fouling of a ceramic MF membrane caused by soluble algal organic matter. Journal of Membrane Science, 493, 683-689.

Zhang, Y., Zhang, J., Xiao, Y., Chang, V.W., Lim, T.-T. 2016. Kinetic and mechanistic investigation of azathioprine degradation in water by $\mathrm{UV}, \mathrm{UV} / \mathrm{H} 2 \mathrm{O} 2$ and UV/persulfate. Chemical Engineering Journal, 302, 526-534. 


\section{Figure Captions}

Figure 1: A schematic diagram of the lab-scale FO system

Figure 2. Impact of pre-treatments on FO organic fouling by ATDE (a) flux performance (b) water recovery (data presented as average \pm standard deviation of duplicate samples)

Figure 3 (a) Excitation emission-matrix spectrum of the untreated ATDE (b) the volumes of EEM spectrum for untreated and various pre-treated ATDE samples.

Figure 4: (a) LC-OCD chromatogram for the ATDE and (b) residual DOC of the different fractions after the pre-treatments as detected by LC-OCD.

Figure 5: Distribution of the (a) reversible and (b) irreversible organic foulants for untreated and pre-treated ATDE samples on the FO membrane with successive filtration 


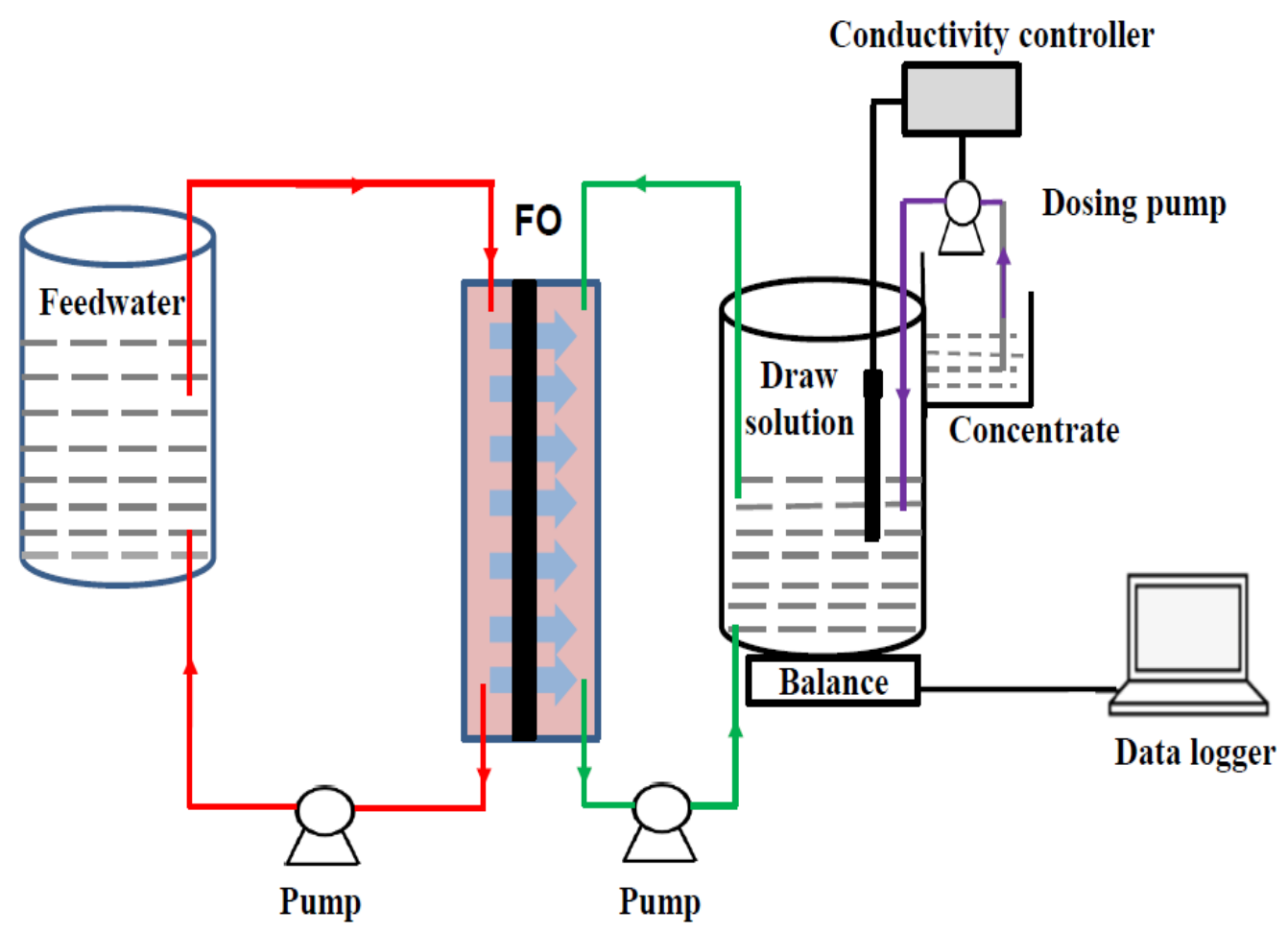

Figure 1 
(a)

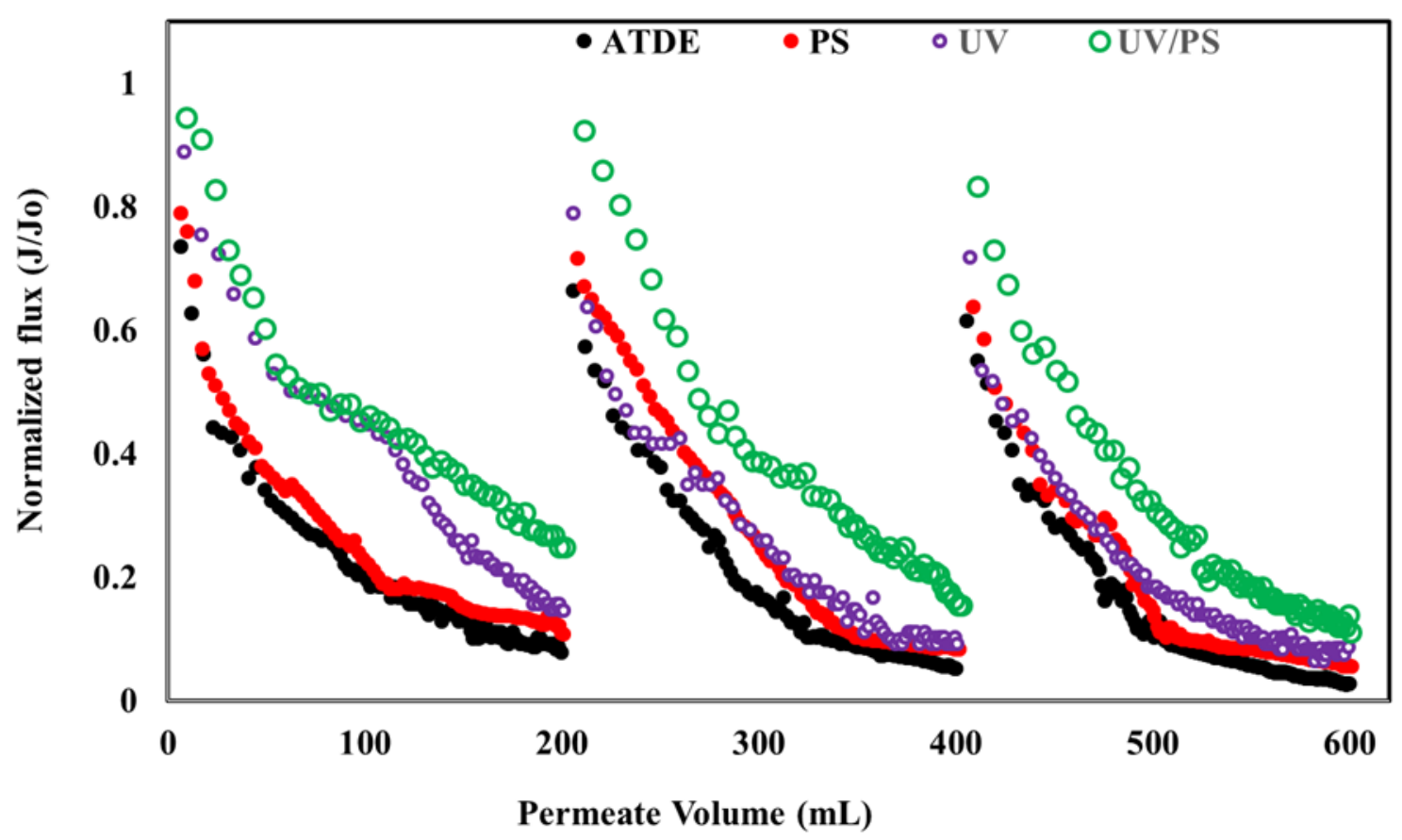

(b)

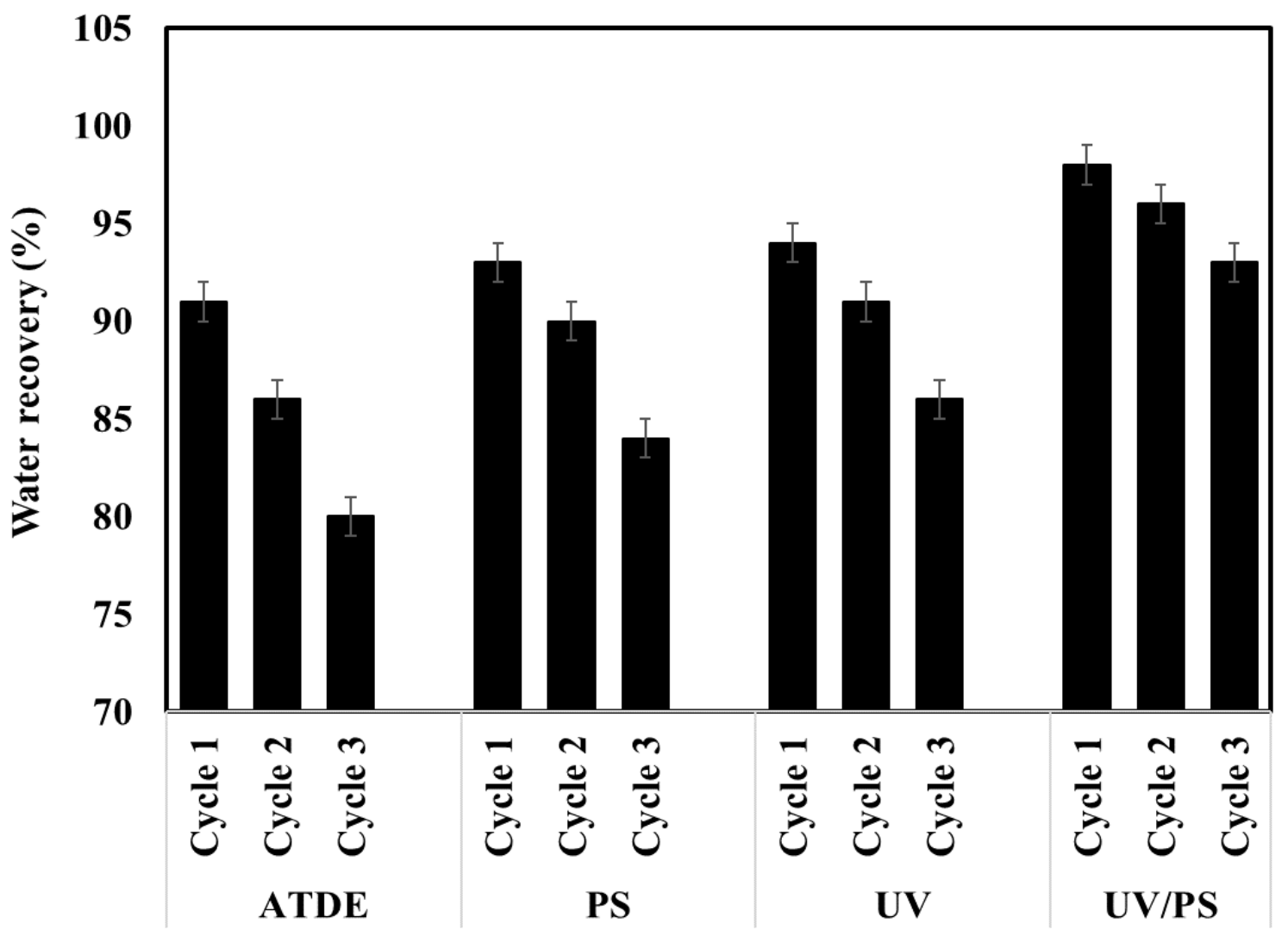

Figure 2 
(a)

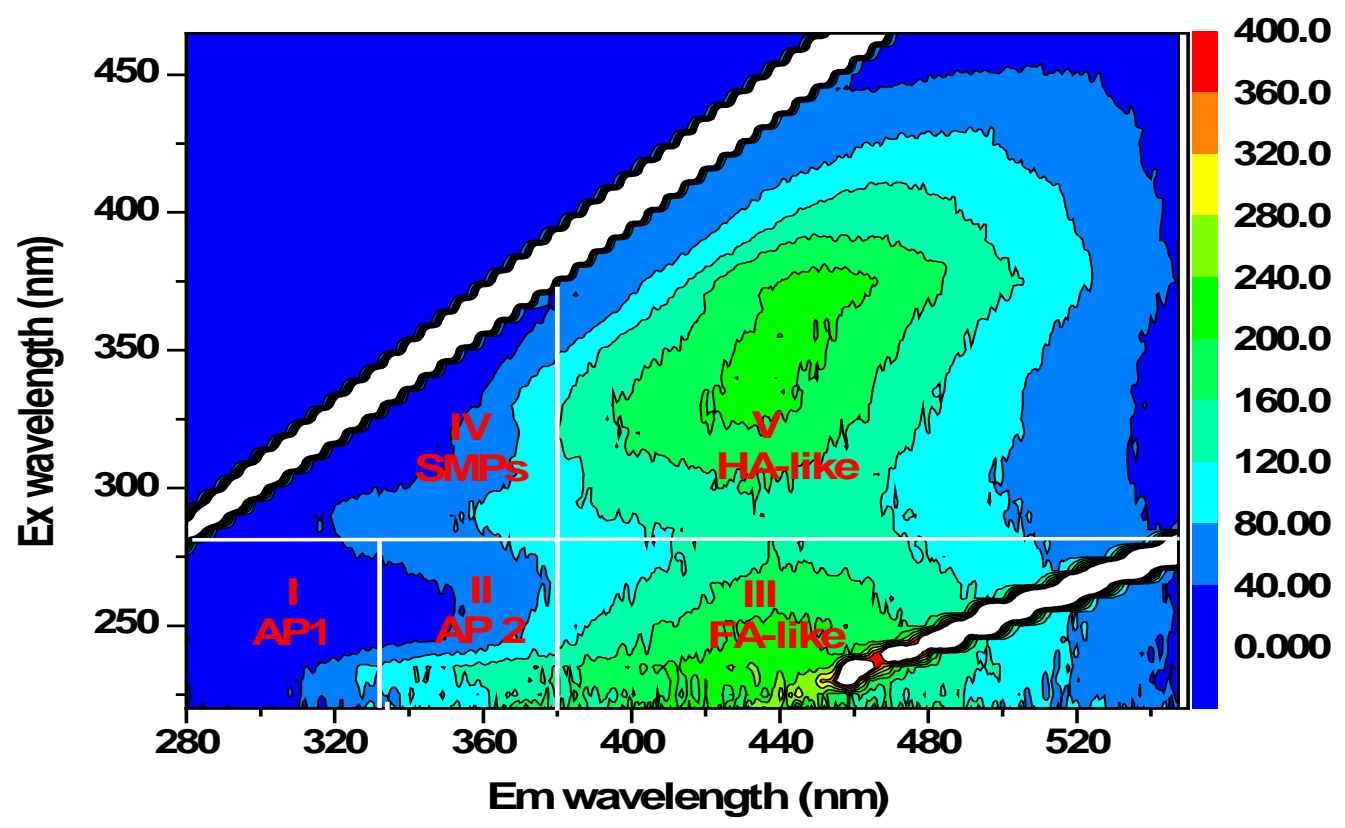

(b)

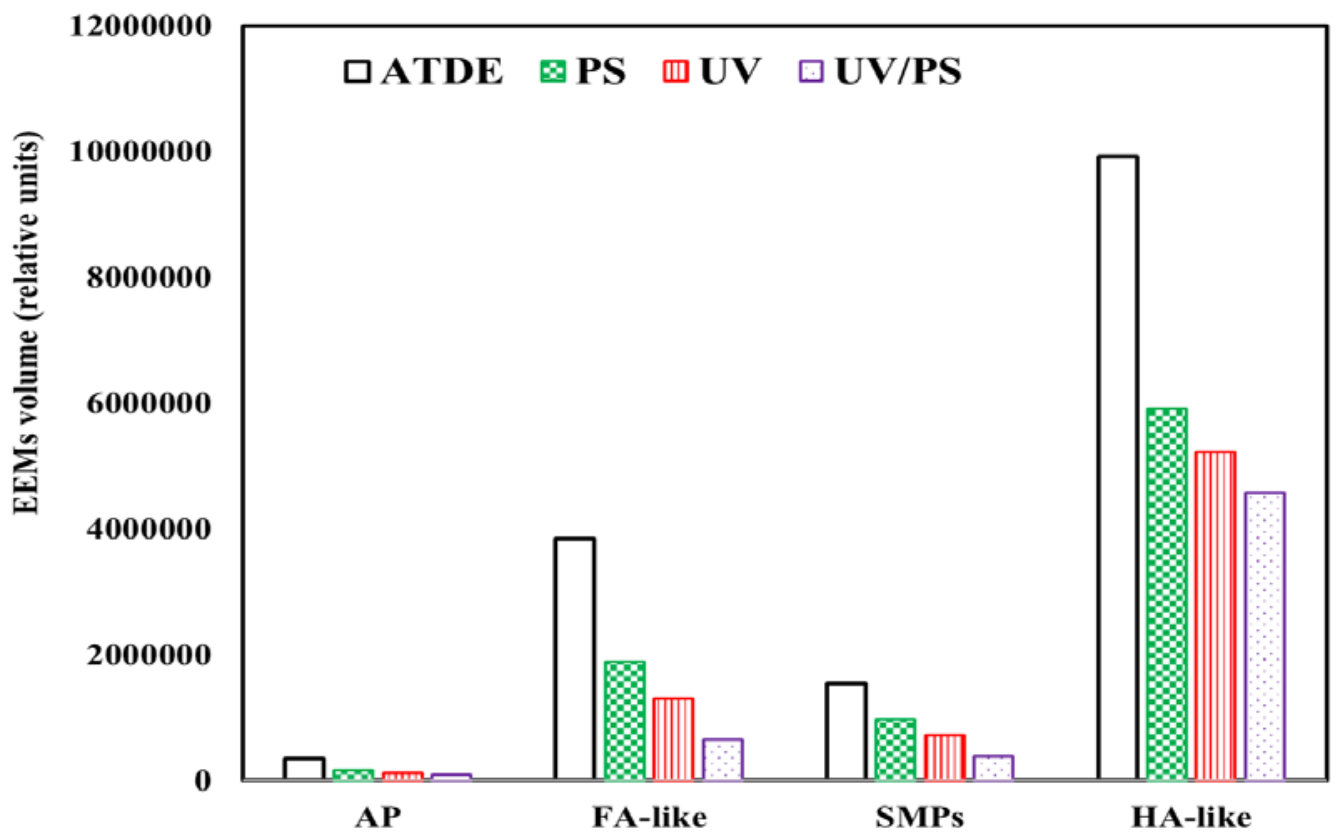

Figure 3 
(a)

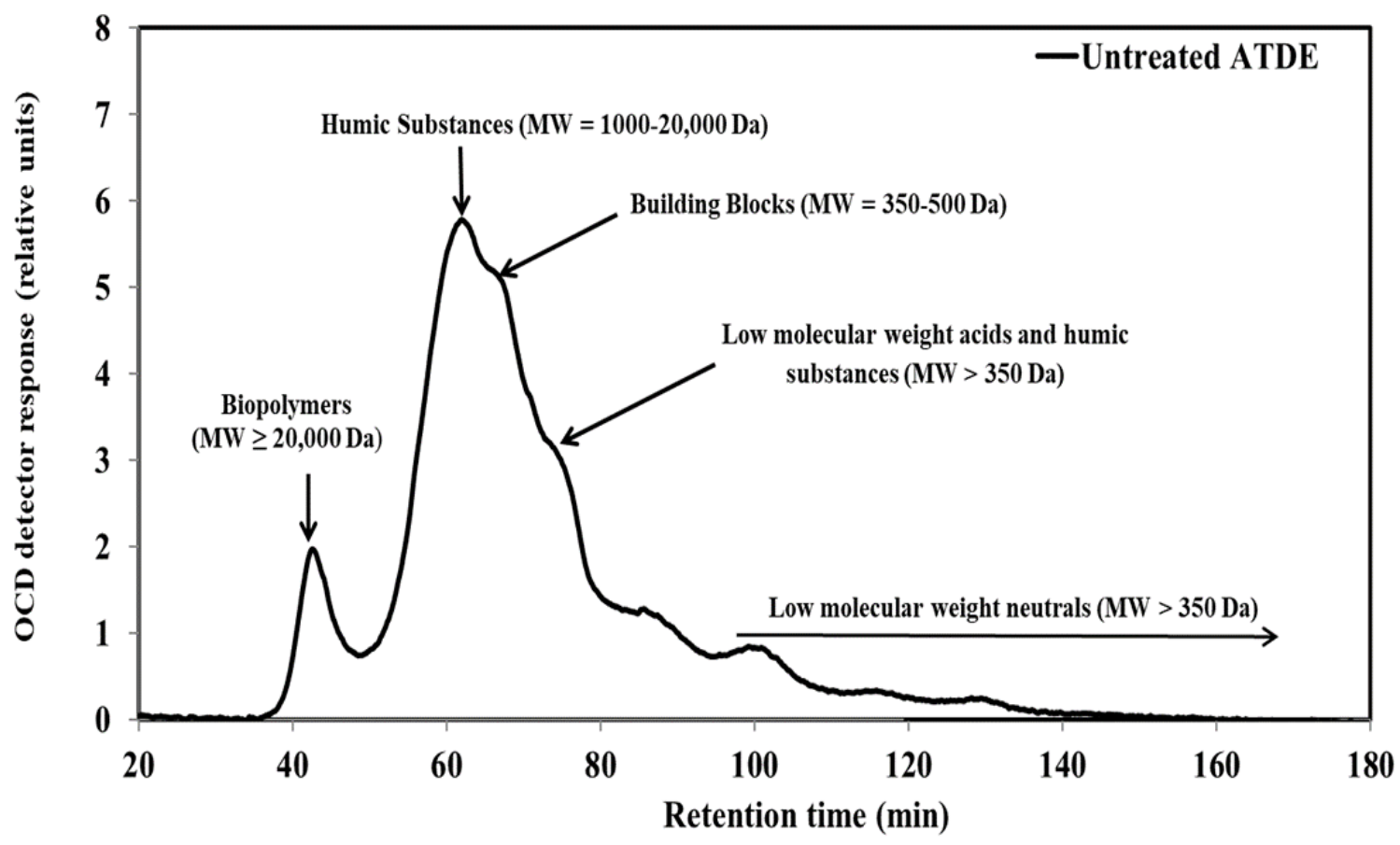

(b)

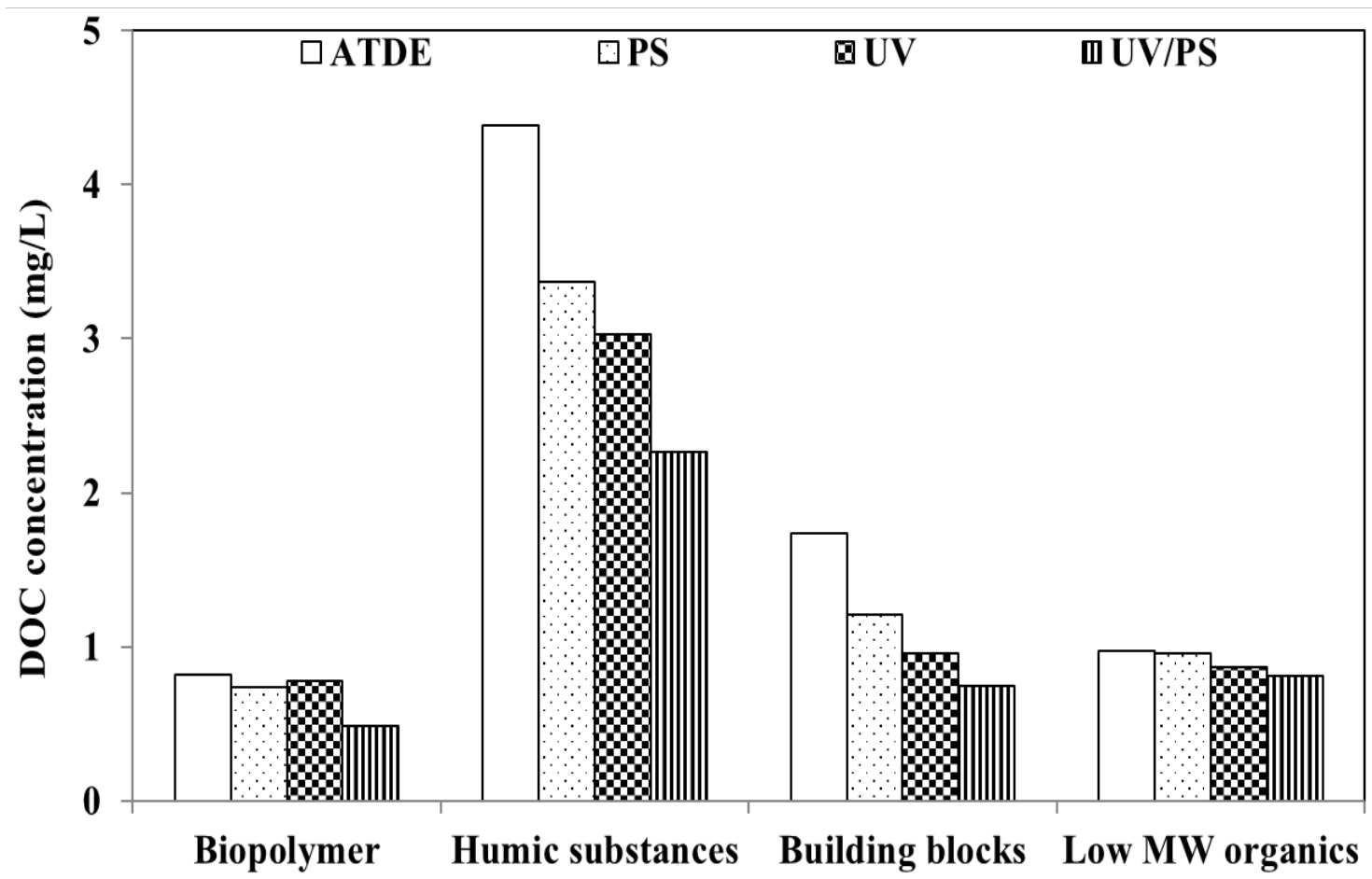

Figure 4 
(a)

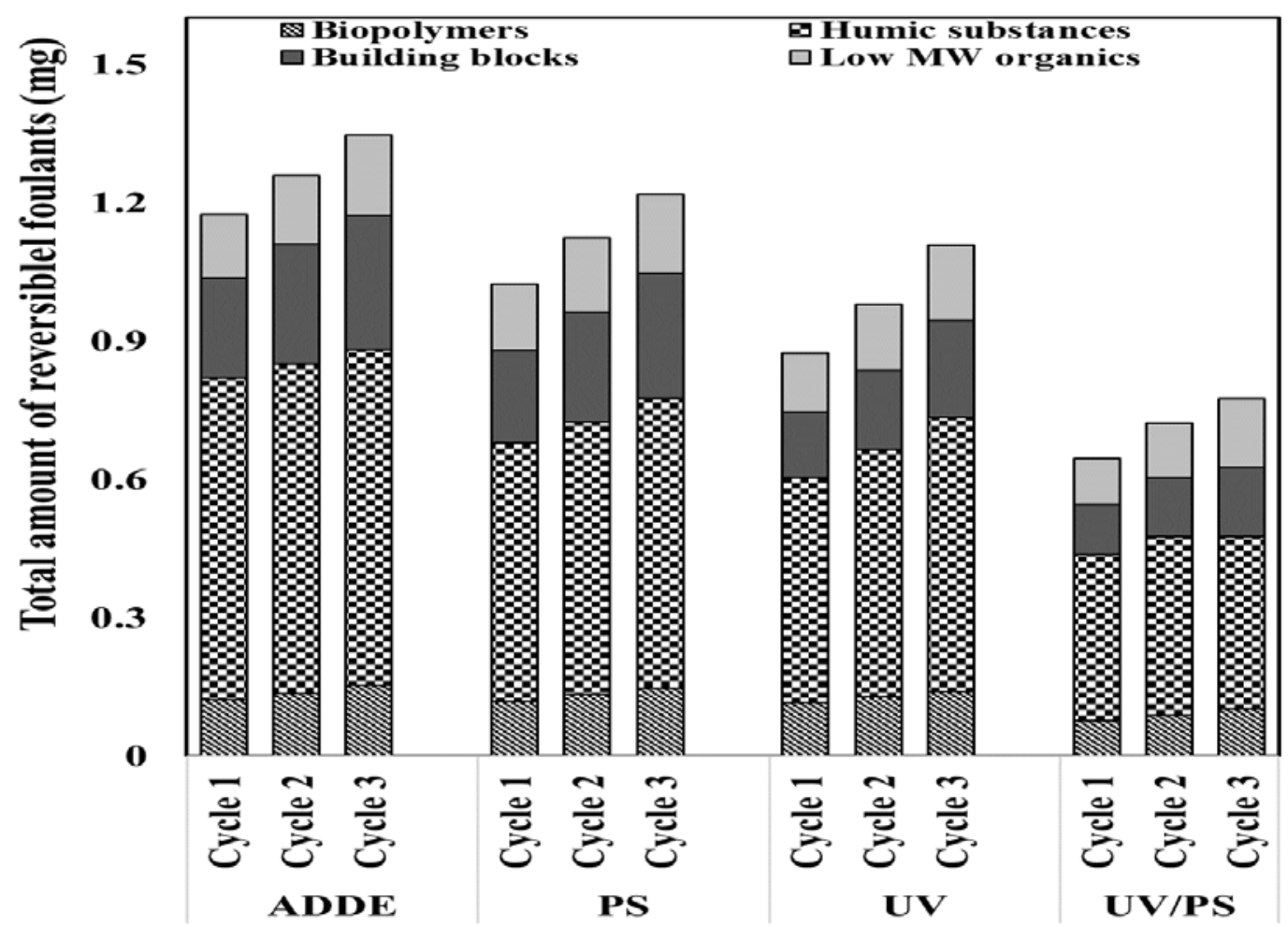

(b)

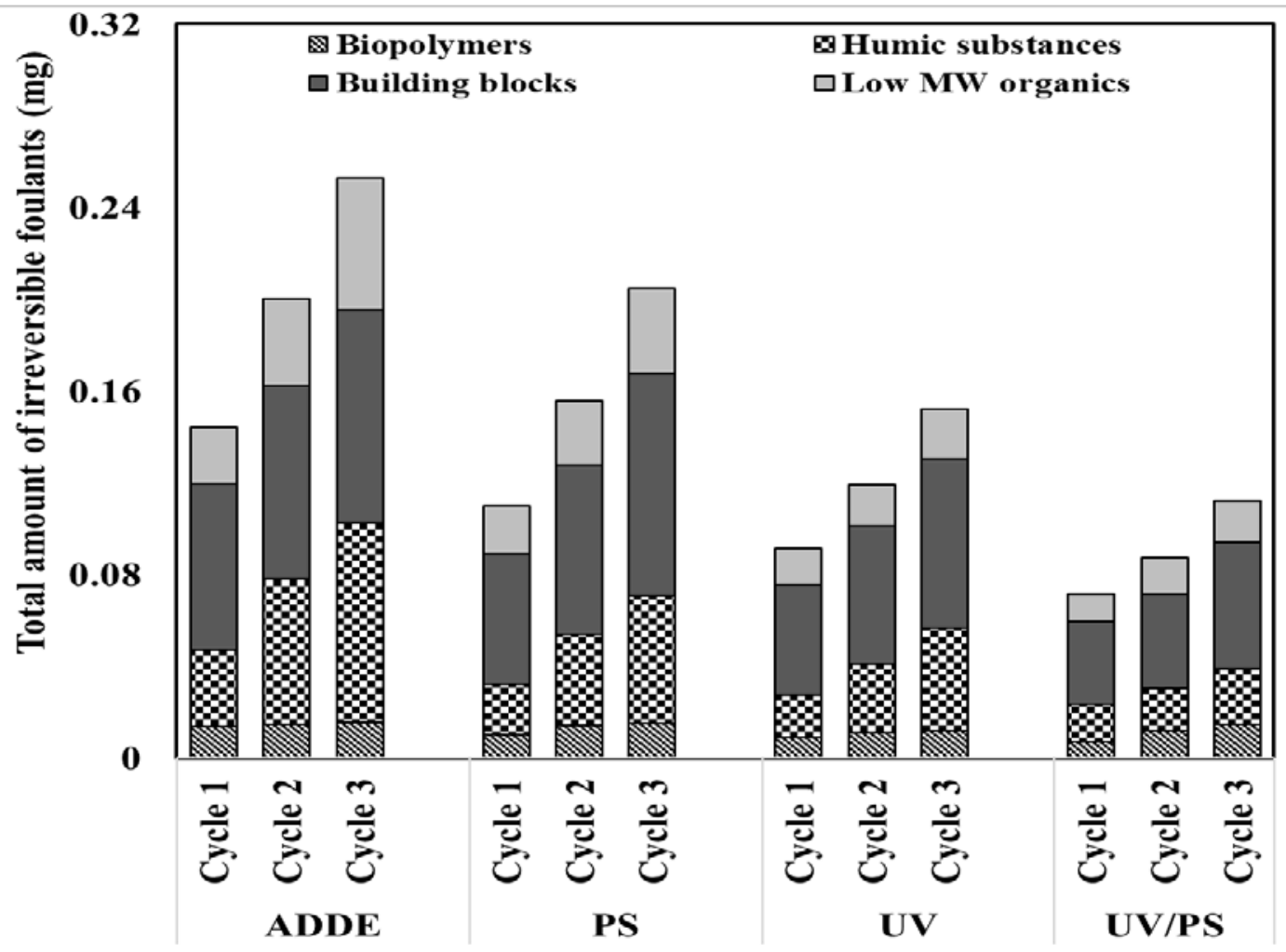

Figure 5 
Table 1: Characteristics of ATDE sample before and after pre-treatments (values indicate average \pm standard deviation of duplicate samples)

\begin{tabular}{lllll}
\hline & ATDE & PS-treated & UV-treated & UV/PS-treated \\
\hline DOC (mg/L) & $54.8 \pm 3.2$ & $49.3 \pm 4.2$ & $46.6 \pm 3.8$ & $38.4 \pm 3.4$ \\
UV $_{254}(/ \mathrm{cm})$ & $1.79 \pm 0.26$ & $1.46 \pm 0.22$ & $1.32 \pm 0.16$ & $1.04 \pm 0.18$ \\
SUVA (L/m.mg) & 3.27 & 2.96 & 2.83 & 2.71 \\
TN & $162 \pm 8$ & $149 \pm 7$ & $146 \pm 6$ & $138 \pm 7$ \\
TP & $112 \pm 3$ & $107 \pm 4$ & $105 \pm 3$ & $98 \pm 3$ \\
\hline
\end{tabular}




\section{Supplementary Information}

Table S1: Key properties of the TFC membrane (Zheng et al., 2018).

\begin{tabular}{ll}
\hline Properties & Value \\
Pore radius $(\mathrm{nm})$ & $0.37 \pm 0.04$ \\
Water permeability coefficient $\left(\mathrm{L} / \mathrm{m}^{2} \mathrm{~h} . \mathrm{bar}\right)$ & $3.2 \pm 0.22$ \\
Salt $(\mathrm{NaCl})$ permeability coefficient $\left(\mathrm{L} / \mathrm{m}^{2} \mathrm{~h}\right)$ & $0.41 \pm 0.01$ \\
Membrane structure parameter $(\mathrm{mm})$ & $0.46 \pm 0.05$ \\
Zeta potential (mV) & -16.2 \\
Contact angle (active layer) $\left(^{\circ}\right)$ & $49.5 \pm 3.4$ \\
\hline
\end{tabular}

